\title{
Langevin Description of Markov Master Equations II: Noise Correlations
}

\author{
P. Hanggi \\ Polytechnic Institute of New York, Department of Physics, Brooklyn, New York, USA
}

Received November 20, 1980; revised version April 27, 1981

In this paper we examine the cumulant properties of generally multiplicative noise of stochastically equivalent stochastic differential equations (SDE) for a given (integro) master equation. For an Ito-SDE we obtain as a necessary consequence that the noise $f_{I}(t)$ possesses a $\delta$-correlated 2-nd order conditioned cumulant $\left\langle f_{I}\left(t_{1}\right) f_{I}\left(t_{2}\right) \mid x\left(t^{*}\right)=x\right\rangle$ if $t^{*} \leqq \max \left\{t_{2}, t_{1}\right\}$. For time points $\left\{t_{1} \leqq t_{2} \ldots \leqq t_{n-1}=t_{n}\right\}$ the conditioned cumulants of $f_{I}(t)$ of order $n>2$ generally contain memory contributions, but vanish if $t_{n-1}<\mathrm{t}_{n}$ and $t^{*} \leqq t_{n}$. These memory terms are not of relevance for the measure of the macroscopic process $x(t)$. Focussing on an alternative non-Ito SDE description we discuss the resulting facts. The character of multiplicative noise is clearly not removable by choosing a different stochastic calculus. The cumulants of order $n>1$ of the noise $f_{N I}(t)$ generally contain memory contributions which are different from the corresponding possibly non-zero (Ito)-memory terms.

\section{Introduction}

The statistical evolution of a macroscopic system can in many situations be modeled reasonably well by a stochastic Markov process $x(t)$. The evolution of the single event probability $p_{t}(x)$ is governed by the master equation. If the increments are of a discontinuous character (jumps) the master equation is of the integro-type with a transition function $W(y \rightarrow x)=W(x, y)$. The master equation describes all the statistical properties. From a principal point of view there is consequently no need to force a Markov process $x(t)$ into a stochastic differential equation (SDE) framework. However, as already pointed out previously [1] (in the following this reference will be denoted by I) and explicitly in [2], the investigation of the underlying noise structure clarifies

* It may be worthwhile pointing out that the sequence of coefficients $\left\{b_{n}\right\}$ in [7] cannot be chosen arbitrarily: The coefficients $\left\{b_{n}\right\}$ must form a sequence of moments. Also, relation (3.26) in [6], which is important in the study of coloured noise phenomena, contains a misprint for a sign. Correct (3.26) reads:

$$
\begin{aligned}
& \frac{\delta z(t)}{\delta f(s)}=b(z(s), s) \\
& +\int_{s}^{\mathrm{r}} d \tau\left\{\frac{\partial}{\partial z(\tau)} a(z(\tau), \tau)+\frac{\partial}{\partial z(\tau)} b(z(\tau), \tau) f(\tau)\right\} \frac{\delta z(\tau)}{\delta f(s)}
\end{aligned}
$$

the involved physics and form of the master equation. The SDE may also simplify calculations of certain statistical quantities. For Fokker-Planck processes those relationships are of course known [3$5,9]$ : The elementary noise source is Gaussian white noise $\xi(t)$ which represents the building stone for more complicated noise as e.g. multiplicative noise yielding non-constant diffusion coefficients. For the case of discontinuous processes it has been demonstated previously $[1,6,7]^{\star}$ that the appropriate elementary noise element is white Poissonian counting noise $\zeta(t ; u)$ with $u$ denoting a fixed jump size. The statistics of $\zeta(t ; u)$ is characterized by its cumulant averages [1]

$$
\langle\zeta(t ; u)\rangle=0
$$

and

$$
\begin{aligned}
& \left\langle\zeta\left(t_{1} ; u\right) \ldots \zeta\left(t_{n} ; u\right)\right\rangle_{c} \\
& =\lambda_{u} \delta\left(t_{1}-t_{2}\right) \ldots \delta\left(t_{n-1}-t_{n}\right) .
\end{aligned}
$$

The index (c) denotes the cumulant average and $\lambda_{u}$ is the Poisson counting parameter which may depend on the jump size $u$. 
Starting from a general master equation the construction of a stochastically equivalent SDE has been presented in I using Ito calculus. In a recent publication [8], Van Kampen put forward a SDE description for the radioactive decay problem. In contrast to the approach in I, where we derived the results by use of an elementary representation for the generally multiplicative noise, Van Kampen elaborates on the cumulant properties of the multiplicative noise. He shows that a third order cumulant average of the noise contains generally memory contributions. This correct result contradicts an expression in (I.5.30c) for the cumulant average.

In this paper we examine in more detail the cumulant properties of generally multiplicative noise occuring in a SDE description of a Markov process; thereby correcting a mistake in (I.5.30c). Loosely speaking one often argues that the temporal evolution of a system is Markovian if and only if the environmental fluctuations are $\delta$-correlated. As it will be shown below, such a statement is correct only if the term "fluctuations" refers to the elementary noise sources $\xi(t)$ and $\zeta(t ; u)^{\star}$. Multiplicative noise represents a generalized [9] macroscopic process and such a multiplicative noise will generally exhibit memory whenever we consider conditioned or unconditioned multi-time moments. As shown in the Appendix this fact holds even for the case of multiplicative continuous noise in a SDE for FokkerPlanck processes.

The outline of the paper is as follows: In Sect. 2 we study the correlation properties of multiplicative noise of an Ito-SDE. In Sect. 3 we present some facts concerning a non-Ito-SDE description of the process $x(t)$.

The main results of the paper can be summarized as follows:

1) Using for a given master equation an Ito-SDE, the noise $f_{I}(t)$ has a $\delta$-correlated 2-nd order conditioned cumulant $\left\langle f_{I}\left(t_{1}\right) f_{I}\left(t_{2}\right) \mid x\left(t^{*}\right)=x\right\rangle_{c}$ for $t^{*} \leqq \max \left\{t_{2}, t_{1}\right\}$. Non-equal time cumulants of order $n>2$ with $\left\{t_{1} \leqq t_{2} \leqq \ldots \leqq t_{n-1}=t_{n}\right\}$, conditioned at time $t^{*} \leqq t_{n}$, generally contain a memory contribution, but vanish if $t_{n-1}<t_{n}$. The highest order singular term is given by a $(n-1)$-dimensional $\delta$-function and the memory terms are of less singular character.

2) The memory terms are not of relevance for the measure of the macroscopic process $x(t)$.

3) For a non-Ito SDE description with noise $f_{N I}(t)$ the conditioned cumulant averages of order $n>1$ generally contain memory contributions which differ

* More generally, strictly additive noise $f(t)$ possesses $\delta$-correlated unconditioned cumulants from the corresponding possibly non-zero Ito-memory contributions. However, the $n$-th order cumulant, $n>1$, of $f_{N I}(t)$ contains as highest order singular part the equal time Ito-cumulant, (2.18), of order $n$, being proportional to the $n$-th KramersMoyal moment $A_{n}(x)$ if $t^{*}=t_{n}$.

\section{Noise Correlations}

The theory in this section is outlined for discontinuous processes (i.e. all variations are of step character). The case of continuous processes is contained implicitly by letting the jump frequency $\lambda_{u}$ go to infinity and the jump size $u$ go to zero such that $\lambda_{u} \cdot u^{2}$ $=$ const. [2]. Given a master equation, we can recast the integro equation

$\dot{p}_{t}(x)=\int W(x, y) p_{t}(y) d y$

into the form of the Kramers-Moyal expansion

$\dot{p}_{t}(x)=\sum_{n=1}^{\infty} \frac{(-1)^{n}}{n !}\left(\frac{\partial}{\partial x}\right)^{n}\left\{A_{n}(x) p_{i}(x)\right\}$.

With an arbitrary function $B(x)$ the SDE

$\dot{x}(t)=B(x(t))+f(t)$

is a stochastically equivalent description of the Markov process in (2.2) if the generally multiplicative noise $f(t)$ satisfies the condition I, II [2]:

I) $\lim _{\Delta \rightarrow 0} \frac{1}{\Delta} \int_{t}^{t+\Delta} d s\langle f(s) \mid x(t)=x\rangle=A_{1}(x)-B(x)$.

Here $\langle\mid x\rangle$ denotes the conditional average. The part $\left(A_{1}(x)-B(x)\right)$ presents the fluctuation induced drift. The higher order conditioned cumulants (denoted by an index c) must obey

II) $\lim _{\Delta \rightarrow 0} \frac{1}{\Delta} \int_{t}^{t+\Delta} \ldots \int_{t}^{t+\Delta} d t_{1} \ldots d t_{n}\left\langle f\left(t_{1}\right) \ldots f\left(t_{n}\right) \mid x(t)=x\right\rangle_{c}$
$=A_{n}(x) \quad n \geqq 2$.

Here the $\left\{A_{n}(x)\right\}$ are the Kramers-Moyal moments in (2.2).

Obviously, with such a formulation the concept of an Ito or non-Ito interpretation does not enter. As long as the integral properties in (2.4), (2.5) hold for the noise, the process $x(t)$ is described stochastically equivalently by the corresponding $\operatorname{SDE}$ (2.3). From a mathematical point of view, any stochastic calculus with

$\lim _{d t \rightarrow 0}\langle d x(t) \mid x(t)=x\rangle / d t=A_{1}(x)$ 
and cumulant averages for the increments $d y(t)$ $=f(t) d t$

$\left\langle(d y(t))^{n} \mid x(t)=x\right\rangle_{c}$

$=A_{n}(x) d t+d t O(d t), \quad n \geqq 2$

yields a consistent description of the Markov process $x(t)$.

The concept of Ito or non-Ito interpretation enters when we give the noise $f(t)$ a more explicit meaning via an elementary representation (interpretation of stochastic integral).

$$
\begin{aligned}
& f(t)=\frac{d y(t)}{d t} \\
& =\lim _{d t \rightarrow 0}\left[\int_{t}^{t+d t} d s \int g(t, x(s), u) \zeta(s ; u) d u\right] / d t .
\end{aligned}
$$

Next we investigate the details of the cumulant properties of stochastically equivalent noise $f_{I}(t)$; i.e. we use for $f(t)$ an elementary representation (2.8) with the right hand side of (2.8) interpreted using Ito rules [1]. Although a general noise is a superposition over all jump sizes of mutually independent elementary noise $\zeta(t ; u)$ we can discuss the memory effects by assuming, for the sake of simplicity only, that $f_{I}(t)$ has the special representation

$f_{I}(t) d t=d y_{I}(t)=g(x(t)) d \eta(t)$

where

$d \eta(t)=\zeta(t ; u) d t$

with $u$ fixed and

$\lambda_{u z}=1$.

If we note the Ito starting point rule [1] in the multiplication in (2.9), implying $g(x(t))$ to be independent of $d \eta(t)$, we find [1]

$\left\langle f_{I}\left(t_{1}\right) \mid x\left(t^{*}\right)=x\right\rangle=0, \quad t_{1} \geqq t^{*}$.

The relation (2.12) is generally not true for $t^{*}>t_{1}$, since for $t^{*}>t_{1}, d \eta\left(t_{1}\right)$ is not independent of $x\left(t^{*}\right)$. Nevertheless, we obtain from (2.12) for the unconditioned average

$\left\langle f_{I}\left(t_{1}\right)\right\rangle=\int\left\langle f_{I}\left(t_{1}\right) \mid x\left(t^{*}\right)=x\right\rangle p_{t^{*}}(x) d x=0$.

Because only conditioned cumulants do enter the discussion of the SDE we will in the following consider only conditional multi-time cumulant averages. Moreover, because the solution $x(t)$ in (2.3) is a functional $\mathscr{F}\left(f(s) ; t_{0} \leqq s \leqq t\right)$ of the noise $f(s)$, we restrict the discussion of conditioned averages to those cases for which the time-point of the condition $t^{*}$ lies between $t_{0} \leqq t^{*} \leqq t_{f}$ with $t_{f}$ being the latest time involved in the expression of the multi-time average. Considering a 2 -nd order average, we obtain in view of (1.2) and (2.11)

$\left\langle\left(d y_{I}\left(t_{1}\right)\right)^{2} \mid x\left(t^{*}\right)=x\right\rangle_{c}$
$=\left\langle g^{2}\left(x\left(t_{1}\right)\right) \mid x\left(t^{*}\right)=x\right\rangle d t_{1}, \quad t_{1} \geqq t^{*}$.

For $t^{*} \leqq \max \left\{t_{2}, t_{1}\right\}, t_{2} \neq t_{1}$ we have

$\left\langle d y_{I}\left(t_{1}\right) d y_{I}\left(t_{2}\right) \mid x\left(t^{*}\right)=x\right\rangle_{c}=0$,

$t_{2} \neq t_{1}, t^{*} \leqq \max \left\{t_{2}, t_{1}\right\}$

(2.14) and (2.15) are combined to give

$$
\begin{aligned}
& \left\langle f_{I}\left(t_{1}\right) f_{I}\left(t_{2}\right) \mid x\left(t^{*}\right)=x\right\rangle_{c} \\
& =\left\langle g^{2}\left(x\left(t_{2}\right)\right) \mid x\left(t^{*}\right)=x\right\rangle \delta\left(t_{1}-t_{2}\right) \quad t^{*} \leqq \max \left\{t_{2}, t_{1}\right\} .
\end{aligned}
$$

An important cumulant average, which enters the discussion of the SDE construction $[1,2]$ is with (1.2) and (2.11)

$$
\begin{aligned}
& \left\langle\left(d y_{1}(t)\right)^{n} \mid x(t)=x\right\rangle_{c}=g^{n}(x)\langle\zeta(t) \ldots \zeta(t)\rangle_{c}(d t)^{n} \\
& =g^{n}(x) d t, \quad n \geqq 2 .
\end{aligned}
$$

In terms of the noise $f_{I}(t),(2.17)$ is recast as

$\lim _{d t \rightarrow 0}\left[(d t)^{n-1}\left\langle f_{I}\left(t_{1}\right) \ldots f_{Y}\left(t_{n}\right) \mid x\left(t^{*}\right)=x\right\rangle_{c}\right]=g^{n}(x)$,

$t_{1}=\ldots=t_{n}=t^{*}, n \geqq 2$.

(2.18) shows that the cumulant $\left\langle f_{I}\left(t_{1}\right) \ldots f_{I}\left(t_{n}\right)\right| x\left(t_{n}\right)$ $=x\rangle, t_{i} \leqq t_{n}$, contains a $(n-1)$-dimensional $\delta$-function and possibly a memory part $m_{n}\left(t_{1}, \ldots, t_{n} ; x t_{n}\right)$

$\left\langle f_{I}\left(t_{1}\right) \ldots f_{I}\left(t_{n}\right) \mid x\left(t_{n}\right)=x\right\rangle_{c}$

$=g^{n}(x) \delta\left(t_{1}-t_{2}\right) \ldots \delta\left(t_{n-1}-t_{n}\right)+m_{n}\left(t_{1}, \ldots, t_{n} ; x, t_{n}\right)$,

$t_{i} \leqq t_{n}, i<n, n \geqq 2$.

In order not to contribute to (2.18), the memory function is of less singular character; i.e. it does not contain a $(n-1)$-dimensional $\delta$-function. Because the averages are symmetric with respect to the timepoints $\left\{t_{1}, \ldots, t_{n}\right\}$ it is sufficient to consider $\left\{t_{1} \leqq t_{2} \leqq \ldots \leqq t_{n-1} \leqq t_{n}\right\}$. With $t^{*} \leqq t_{n}$ we obtain more generally

$$
\begin{aligned}
& \left\langle f_{I}\left(t_{1}\right) \ldots f_{I}\left(t_{n}\right) \mid x\left(t^{*}\right)=x\right\rangle_{c} \\
& =\left\langle g^{n}\left(x\left(t_{n}\right)\right) \mid x\left(t^{*}\right)=x\right\rangle \delta\left(t_{1}-t_{2}\right) \ldots \delta\left(t_{n-1}-t_{n}\right) \\
& +m_{n}\left(t_{1}, \ldots, t_{n} ; x, t^{*}\right), \\
& t_{1} \leqq \ldots \leqq t_{n}, t^{*} \leqq t_{n}, n \geqq 2 .
\end{aligned}
$$


From (2.19), (2.18) we find

$\lim _{d t \rightarrow 0}\left[(d t)^{n-1} m_{n}\left(t_{1}, \ldots, t_{n} ; x, t^{*}\right)\right]=0$

and from (2.16)

$m_{2}\left(t_{1}, t_{2} ; x, t^{*}\right)=0, \quad t^{*} \leqq \max \left\{t_{2}, t_{1}\right\}$.

For the sequence

$t_{1} \leqq t_{2} \leqq \ldots \leqq t_{n-1}<t_{n} ; \quad t^{*} \leqq t_{n}$

the corresponding cumulant reads

$\left\langle f_{I}\left(t_{1}\right) \ldots f_{I}\left(t_{n}\right) \mid x\left(t^{*}\right)=x\right\rangle=0$

so that

$m_{n}\left(t_{1}, \ldots, t_{n} ; x, t^{*}\right)=0, \quad t_{1} \leqq \ldots \leqq t_{n-1}<t_{n}, t^{*} \leqq t_{n}$.

This result follows from the fact that in a conditioned moment of order $i \leqq n$ containing the random variable $d \eta\left(t_{n}\right)$ which is of a vanishing average, $d \eta\left(t_{n}\right)$ is independent from the rest. Thus, each moment term in the corresponding cumulant representation is multiplied by zero. This fact explicitly exhibits the properties of the Ito-calculus for $f_{l}(t)$ (martingale property).

The result (2.24) is generally not true if $t_{n-1}=t_{n}$. For example, we find for $t_{1}<t_{2}=t_{3}, t^{*} \leqq t_{3}$ by use of the expression for the third cumulant [10]

$$
\begin{aligned}
& \left\langle d y_{1}\left(t_{1}\right)\left(d y\left(t_{2}\right)\right)^{2} \mid x\left(t^{*}\right)=x\right\rangle_{c} \\
& =\left\langle g\left(x\left(t_{1}\right)\right) g^{2}\left(x\left(t_{2}\right)\right) d \eta\left(t_{1}\right) \mid x\left(t^{*}\right)=x\right\rangle d t .
\end{aligned}
$$

Close inspection of (2.25a) on the intervals ( $\left[t_{0}, t_{1}\right]$; $\left.\left[t_{1}, t_{1}+d t\right] ;\left[t_{1}+d t, t_{2}\right]\right)$ reveals the structure

$$
\begin{aligned}
& \lim _{d t \rightarrow 0}\left[\frac{1}{d t}\left\langle g\left(x\left(t_{1}\right)\right) g^{2}\left(x\left(t_{2}\right)\right) d \eta\left(t_{1}\right) \mid x\left(t^{*}\right)=x\right\rangle\right] \\
& =\lim _{d t \rightarrow 0}\left[d t m_{3}\left(t_{1}, t_{2}=t_{3} ; x, t^{*}\right)\right] \\
& \equiv h\left(t_{1}, t_{2} ; x t^{*}\right) \neq 0 .
\end{aligned}
$$

The memory in $(2.25 \mathrm{~b}$ ) (as well as in (2.19)) results from the fact that the increment $d \eta\left(t_{1}\right)$ is not independent from nonconstant $g^{2}\left(x\left(t_{2}\right)\right), t_{2}>t_{1}$. However, cumulants of the type in (2.25) do not contribute to (2.4), (2.5) (derivation of corresponding master equation).

Only (2.18) does contribute in (2.4), (2.5). In other words, the memory terms are not of relevance for the measure of the corresponding macroscopic process $x(t)$.

Moreover, the results in this section, in particular (2.16) and (2.25), are consistent with Van Kampens' explicit calculations (up to order $n=3$ ) for the cumulant averages for the noise $f_{I}(t)$ of the radioactive decay process [8].

\section{Ito Versus Non-Ito}

Interpreting the elementary representation (2.9) according to a non-Ito calculus we obtain different noise $f_{N I}(t) \neq f_{I}(t)$ with generally different results for corresponding cumulant averages; in particular different results for corresponding memory terms. (For Fokker-Planck processes see the Appendix.) In virtue of $(2.7)$ however, the $n$-th order conditioned cumulant of the noise $f_{N I}(t)$ of a non-Ito-SDE for a given master equation (2.1) must contain the highest order singular term $A_{n}(x) \delta\left(t_{1}-t_{2}\right) \ldots \delta\left(t_{n-1}-t_{n}\right)$, $n \geqq 2, t^{*}=t_{n}$.

In conclusion, the character of multiplicative noise, reflected by non-constant Kramers-Moyal moments $A_{n}(x), n \geqq 2$, is clearly not removed by choosing a different stochastic calculus. However, as shown in [1], (see the Ito-Stratonovitch calculation in Sect. 6 of [1]) the coupling function $g,(2.8)$, is generally sensitive to a change of the stochastic calculus. This is in contrast to the Fokker-Planck case where only the drift expression in the corresponding stochastically equivalent SDE becomes modified when we choose a different stochastic calculus. (Note that with a nonconstant $g$ in a SDE for a discontinuous process we would obtain different Kramers-Moyal moments of order $n \geqq 2$ by choosing different stochastic calculi.) The Ito calculus possesses a particularly simple relationship between coupling function $g$ and Kramers-Moyal moment (see I.5.17).

\section{Appendix}

For a Fokker-Planck process modeled by the stochastically equivalent Ito-SDE $\left(\xi(t)==\frac{d w(t)}{d t}\right.$ standard Gaussian white noise)

Ito: $\dot{x}(t)=b(x(t))+g(x(t)) \xi(t)$

$=b(x(t))+f_{1}(t)$

the corresponding stochastically equivalent Stratonovitch SDE reads $[3-5,9]$

$\dot{x}(t)=b(x(t))-\frac{1}{2} g(x(t)) g^{\prime}(x(t))+g(x(t)) \circ \xi(t)$.

Hereby the increment is interpreted using Stratonovitch rules; i.e. $d y_{S}(t)=g(x(t)) \circ \xi(t)$ (o denotes Stratonovitch multiplication rule). (A.3) is recast into the 
form (2.3)

$\dot{x}(t)=B(x(t))+f_{S}(t)$

with the Stratonovitch noise $f_{\mathrm{S}}(t)$

$f_{S}(t)=g(x(t)) \circ \xi(t)$.

Evaluating the conditional average, $t_{1} \leqq t_{2}=t^{*}$

$\left\langle f_{S}\left(t_{1}\right) f_{S}\left(t_{2}\right) \mid x\left(t_{2}\right)=x\right\rangle$

we obtain

$\left\langle f_{S}\left(t_{1}\right) f_{S}\left(t_{2}\right) \mid x\left(t_{2}\right)=x\right\rangle=g^{2}(x) \delta\left(t_{1}-t_{2}\right)$

$+\frac{1}{4}\left\langle g\left(x\left(t_{1}\right)\right) g^{\prime}\left(x\left(t_{1}\right)\right) g\left(x\left(t_{2}\right)\right) g^{\prime}\left(x\left(t_{2}\right)\right) \mid x\left(t_{2}\right)=x\right\rangle$.

(A.7) contains memory. The first term on the right hand side just gives the Ito result. Observing (A.3) and (A.7) we find for the 2-nd cumulant

$$
\begin{aligned}
& \left\langle f_{S}\left(t_{1}\right) f_{S}\left(t_{2}\right) \mid x\left(t_{2}\right)=x\right\rangle_{c}=g^{2}(x) \delta\left(t_{1}-t_{2}\right) \\
& +\frac{1}{4}\left\langle g\left(x\left(t_{1}\right)\right) g^{\prime}\left(x\left(t_{1}\right)\right) g\left(x\left(t_{2}\right)\right) g^{\prime}\left(x\left(t_{2}\right)\right) \mid x\left(t_{2}\right)=x\right\rangle \\
& -\frac{1}{4}\left\langle g\left(x\left(t_{1}\right)\right) g^{\prime}\left(x\left(t_{1}\right)\right) \mid x\left(t_{2}\right)=x\right\rangle \\
& \cdot\left\langle g\left(x\left(t_{2}\right)\right) g^{\prime}\left(x\left(t_{2}\right)\right) \mid x\left(t_{2}\right)=x\right\rangle .
\end{aligned}
$$

Higher Ito-cumulants generally also contain memory terms. For example, with $t^{*} \leqq t_{1}<t_{2}=t_{3}$ we obtain for the Ito noise $d y_{I}(t)=g(x(t)) \xi(t)$

$$
\begin{aligned}
& \left\langle d y_{I}\left(t_{1}\right)\left[d y_{I}\left(t_{2}\right)\right]^{2} \mid x\left(t^{*}\right)=x\right\rangle \\
& =\left\langle g\left(x\left(t_{1}\right)\right) g^{2}\left(x\left(t_{2}\right)\right)\left[w\left(t_{1}+d t\right)-w\left(t_{1}\right)\right] \mid x\left(t^{*}\right)=x\right\rangle d t \\
& =\sim(d t)^{2} .
\end{aligned}
$$

\section{References}

1. Hanggi, P.: Z. Phys. B - Condensed Matter 36, 271 (1980)

2. Hanggi, P., Shuler, K.E.S., Oppenheim, I.: Physica 107A, 143 (1981)

3. Stratonovitch, R.L.: SIAM J. Control 4, 362 (1966)

4. Graham, R.: Z. Phys. B - Condensed Matter 26, 397 (1977)

5. Ryter, D.: Z. Phys. B - Condensed Matter 30, 219 (1978)

6. Hanggi, P.: Z. Phys. B - Condensed Matter 31, 407 (1978)

7. Bedeaux, D.: Phys. Lett. 62 A, 10 (1977)

8. Van Kampen, N.G.: Phys. Lett. 76 A, 104 (1980)

9. Arnold, L.: Stochastic Differential Equations, New York: J. Wiley 1974

10. Kubo, R.: J. Phys. Soc. Jpn. 17, 1100 (1962)

P. Hanggi

Polytechnic Institute of New York

Department Physics

333 Jay Street

Brooklyn, NY 11201

USA 